\title{
A comparative assessment of the impact of computer assisted learning of knowledge and skill based competencies among undergraduate students in a medical college in South India
}

\author{
Jeyalalitha Rathinam*, Divakar R., Subahan S. P., Preethi A., Vanitha G., Punitha R.
}

Department of Pharmacology, Govt. Stanley Medical College, Chennai, Tamilnadu, India

Received: 06 March 2018

Revised: 12 March 2018

Accepted: 15 March 2018

*Correspondence to: Dr. Jeyalalitha Rathinam, Email: jeya0909@gmail.com

Copyright: (C) the author(s), publisher and licensee Medip Academy. This is an openaccess article distributed under the terms of the Creative Commons Attribution NonCommercial License, which permits unrestricted noncommercial use, distribution, and reproduction in any medium, provided the original work is properly cited.

\begin{abstract}
Background: Pharmacology has always been one subject ultimatum and a cornerstone in building up competency, based on applied therapeutics among medical graduates in prescribing and practicing skills. A focus on an integrated strategical approach towards teaching pharmacology for better proficiency in achieving clinical skills is mandatory. Thus, this study has been done to assess the impact and retainment of overall knowledge gained on various domains, based on a revised method of teaching in pharmacology.

Methods: An observational, questionnaire based comparative study was done in a medical college, in Chennai. The study included two group of students, one who have completed their pharmacology course based on the revised pattern of teaching with a comparative group of students who have not been exposed to the revised pattern of teaching. A feedback survey was also done.

Results: The study results indicated that the average scoring based on the questionnaire was $84 \%$ with the students who have been exposed to revised pattern of teaching in comparison to the average scoring of $70 \%$ with the students who have not been exposed to the new pattern of teaching. The average scoring of the students who were found to agree to several questions on the new method of teaching was $74 \%$ and $22 \%$ of the students were found to disagree. $4 \%$ of students were non responders.

Conclusions: The ultimate improvement in the outcome of health care education can be achieved through appropriate skill building through a simple strategical modification of the regular teaching pattern with an integrated approach involving pharmacologists and clinicians. A refresher course in pharmacology in the final year curriculum and CRRI involving pharmacologists is mandatory.
\end{abstract}

Keywords: Impact and retainment of knowledge, Must know therapeutics, Rational therapeutics, Small group teaching

\section{INTRODUCTION}

Health for all is a national goal. This goal has been aimed to be achieved through several measures. Improvements in medical education and training is one among the foremost areas of concern so as to produce competent "Physicians of First Contact". Ethical concerns with the use of animals has led to newer trends in teaching drug effects through computer simulated learning and there has been a much ado about alternatives to animal experiments rather than a focus on a different strategical approach towards teaching the must know aspects in pharmacology. Improvement of the existing curriculum towards meeting the demands of future quality health care through restructuring of current medical educational pattern has been proposed. Pharmacology integrates almost each and every field of medicine and there is no area of therapeutic practice which does not handle a pharmacological tool in its discipline. The numerous and vast introduction of newer class of wide variety of drugs at a fast pace makes the mastery of the 
subject highly challenging. The competency building measures at an undergraduate level involves, a sound knowledge on various must know drugs useful in day to day clinical practice for the various must know disorders, adequate attainment of knowledge on clinically relevant drug-drug interactions, appropriate routes and dosing schedules, standard therapeutic protocols with appropriate interpretational and interventional skills.

The World Health Organization, in its publication "Teacher's Guide to good prescribing," advocates the need for outcome-based learning in pharmacology with a structured program, planned well in advance concentrating on practice, using disease centered teaching learning activities rather than on theory. ${ }^{1}$ This manual provides a normative model for therapeutic reasoning and prescribing and provides a six-step guide to the process of rational prescribing that will be necessary throughout the clinical career of the medical student. ${ }^{2}$ Clinical pharmacologists play an important role in the development of these skills by teaching clinical pharmacology and therapeutics to undergraduate medical students. ${ }^{2}$ The rational prescribing of drugs is an essential skill of medical doctors.

Teaching pharmacology is a challenging task with most students considering it a dry subject, especially students who are not exposed to clinical applications of the same.,

At present the conventional method of teaching pharmacology involves didactic lectures in large groups, tutorials, chart based practical sessions and computer simulated animal experiments. The awareness on alternatives to animal experiments is widespread and various computer aided packages are available in almost all the medical schools. But teaching and learning pharmacology relevant to the ultimate competency building doesn't alone involve an alternative technique to animal experimentation since animal experiments focus only on a meagre area of knowledge development rather than on all critical areas of therapeutics. Also the outcome of health care education should be directed to ultimate and appropriate skill building and not merely emphasize on acquisition of knowledge alone.

In order to emphasize the need for a systematic approach towards redefining and restructuring of the current curriculum at various levels through an integrated approach, this study has been done with the aim of assessing the impact of small group teaching through computer assisted learning with a set of revised preplanned modules of various must know topics on critical areas of therapeutics and show how skills at the para clinical level, with the goal of stepwise competency building at early clinical years among undergraduate students.

\section{METHODS}

An observational, questionnaire based comparative study was done in a tertiary care hospital in Chennai. The study participants were the undergraduate students of final year course and CRRI. A total of 242 students participated in the study ,150 from the final year and 142 from CRRI. The first group of students were preliminarily taught a revised pattern of pharmacology in the second year course. The same students were included in the study when they were in the end of the final year curriculum to assess the impact and retainment of knowledge gained. A second group of students from the CRRI who have under gone the conventional method of teaching during the second year pharmacology curriculum and who have not been exposed to the revised CAL were included as a comparative group in the study. Informed consent was obtained from all the participants. The study protocol was approved by the institutional ethics committee.

A questionnaire based survey on applied therapeutics with appropriate scoring was used to assess the overall knowledge gained by the students. A readymade questionnaire based on knowledge and skill was prepared as per the CAL methods on various must know aspects from the revised practical teaching learning methods during the second year pharmacology curriculum. All the students were handed over the questionnaire at the same time and the test was conducted over a period of one hour for 50 questions.

The second year revised teaching pattern, included a small group teaching methodology through computer assisted learning. A preplanned schedule of computer assisted learning modes based on knowledge, skill, communication and attitude building competencies were designed with appropriate tasks at the end of each lesson plan. Small group discussions with specific learning objectives on general pharmacology topics, animal experimentation techniques, drug administration skills, clinical pharmacology exercises and various applied aspects on must know topics on therapeutics were appropriately planned and executed during the regular teaching hours after an approval from the academic committee. The students were divided into small groups of 12 per computer. Each small group had a facilitator. The CAL based small group sessions included a simple introductory frame, a learning frame and a task frame without involving any expensive simulated packages, together with video techniques on appropriate lessons, drug administration skills with side by side manikin demonstrations and hands on training along with appropriate objective structured practical examination stations. A feedback form was also obtained from the students based on the Likert scale of assessment.

\section{RESULTS}

The filled in questionnaire was suitably scored and graded. The validity of the CAL and revised practical teaching learning methods during the second year pharmacology curriculum was assessed and compared with respect to the two group of students. The feed back questionnaire was also assessed. 
A total number of 242 students participated in the study. 150 students belonged to the revised pattern of teaching and 142 students were from the regular pattern of curriculum. The scoring was divided into 3 ranges, of students who scored more than $80 \%, 50-79 \%$ and less than $50 \%$. The number of students who obtained a pass scoring of 50 and above, was found to be $84 \%$ with the revised group and $70 \%$ with the regular pattern who have not been exposed to the new pattern of teaching. Students who scored below 50 marks was found to be $16 \%$ with the revised group and $30 \%$ with the regular pattern. A feedback questionnaire survey was also conducted. The average scoring of the students who were found to agree to several questions on the new method of teaching was $74 \%$ and $22 \%$ of the students were found to disagree. $4 \%$ of students were non responders. $70 \%$ found it interesting, $74 \%$ found the lessons to be well planned, $74 \%$ felt that it covered the various must know aspects, $66 \%$ felt that it helped in the performance of theory exams and $66 \%$ felt that it helped in relating knowledge to practice while 52\% felt that it helped in improving their verbal communication skills. $81 \%$ of the students agreed that it helped in clarifying doubts better than lecture classes, $83 \%$ felt that it helped in the way of approach to patients while $89 \%$ agreed that it helped in performing well in the practical exams. Totally $85 \%$ of the students agreed to the usefulness of the method.

\section{Table 1: Performance assessment.}

\begin{tabular}{|c|c|c|c|}
\hline \multicolumn{4}{|c|}{ Performance score range $(\%)$} \\
\hline $\begin{array}{l}\text { Teaching } \\
\text { method }\end{array}$ & $>80$ & $50-79$ & $<50$ \\
\hline Revised [Rv] & $7(4.66)$ & $119(79.33)$ & $24(16)$ \\
\hline Regular [Rg] & $2(1.4)$ & $98(69.01)$ & $42(29.57)$ \\
\hline
\end{tabular}

Rv: $n=150 ; \operatorname{Rg}: n=142$

Table 2: Feedback questionnaire [n=150 (\%)].

\begin{tabular}{|lllllll|}
\hline & Feedback questions & $\begin{array}{l}\text { strongly } \\
\text { agree }\end{array}$ & agree & disagree & $\begin{array}{c}\text { strongly } \\
\text { disagree }\end{array}$ & $\begin{array}{l}\text { Non } \\
\text { responders }\end{array}$ \\
\hline 1. & $\begin{array}{l}\text { The small group teaching pattern } \\
\text { was interesting }\end{array}$ & $14(9.33)$ & $92(61.33)$ & $26(17.33)$ & $10(6.66)$ & $8(5.33)$ \\
\hline 2. & The sessions were well planned & $22(14.66)$ & $89(59.33)$ & $27(18)$ & $10(6.66)$ & $2(1.33)$ \\
\hline 3. & $\begin{array}{l}\text { Helped in clarifying doubt better } \\
\text { than lecture classes }\end{array}$ & $20(13.33)$ & $101(67.33)$ & $20(13.33)$ & $6(4)$ & $3(2)$ \\
\hline 4. & $\begin{array}{l}\text { Covered must know aspects in } \\
\text { applied therapeutics }\end{array}$ & $24(16)$ & $87(58)$ & $24(16)$ & $11(7.33)$ & $4(2.66)$ \\
\hline 5. & $\begin{array}{l}\text { Helped in scoring well in the } \\
\text { theory exam }\end{array}$ & $21(14)$ & $78(52)$ & $26(17.33)$ & $21(14)$ & $4(2.66)$ \\
\hline 6. & $\begin{array}{l}\text { Helped in scoring well in the } \\
\text { practical exam }\end{array}$ & $30(20)$ & $104(69.33)$ & $12(8)$ & $2(1.33)$ & $2(1.33)$ \\
\hline 7. & $\begin{array}{l}\text { Helped relate knowledge to } \\
\text { clinical practice }\end{array}$ & $19(12.66)$ & $80(53.33)$ & $21(14)$ & $19(12.66)$ & $11(7.33)$ \\
\hline 8. & $\begin{array}{l}\text { Helped in improving verbal } \\
\text { communication skill }\end{array}$ & $10(6.66)$ & $68(45.33)$ & $56(37.33)$ & $4(2.66)$ & $12(8)$ \\
\hline 9. & $\begin{array}{l}\text { Helped in way of approach to } \\
\text { patients }\end{array}$ & $27(18)$ & $98(65.33)$ & $18(12)$ & $3(2)$ & $4(2.66)$ \\
\hline 10. & $\begin{array}{l}\text { Useful and helped in better } \\
\text { understanding of pharmacology }\end{array}$ & $31(20.66)$ & $96(64)$ & $12(8)$ & $8(5.33)$ & $3(2)$ \\
\hline
\end{tabular}

\section{DISCUSSION}

The study has been done utilizing the method of small group teaching with simple computer aided classes on various must know, clinically oriented topics. Several studies have already stated the usefulness of computer assisted learning and small group teaching. Computerbased learning is often regarded as a teaching method in its own right but in fact it is simply a delivery mechanism capable of being used to provide access to a variety of learning aids involving many different teaching methods. ${ }^{5}$
The most important advantage of CAL is that they meet the majority of the learning objectives. There is supporting evidence from many medical schools that CAL is the best suitable alternative to practical laboratory classes and successfully meets the learning objectives of the sessions. ${ }^{6,7}$ The effectiveness of small group teaching against didactic lectures has also been well documented. ${ }^{8,9}$

Studies have pointed out the importance and usefulness of teaching modules, for better understanding and retainment of knowledge. It is insufficient to just develop computerbased learning material available to students. Like a 
laboratory class, it must be fully integrated into the modules to obtain the desired benefits. ${ }^{10}$

This study has been done on students who have completed their pharmacology course and who were in the final year curriculum so as to assess the impact and retainment of the knowledge gained on critical areas of therapeutic skill development learnt during the pharmacology course. The study results indicates, that the scoring obtained by the students who attended the revised method of teaching is better than the scoring obtained by the students who had undergone regular pattern of teaching. The CAL based small group teaching enabled the students to concentrate and comprehend the sessions better with motivated interactions from the facilitator.

Moreover, the feedback questionnaire survey revealed that the students found the classes livelier and interesting than the continuous didactic lectures in large groups especially in the afternoon post lunch sessions. $70 \%$ of the students felt the classes were interesting. Even though there was a better interaction with the facilitators and the slow learners were found to be better involved in comparison to fast learners, only $52 \%$ of the students felt that it has helped them with their verbal communication skills. The undergraduate students have different medium of education in the precollege years and thus it mandates certain essential measures to improve their proficiency in understanding and communication. This can overcome barriers on therapeutic interpretation and execution skills.

The various must know aspects on critical areas of clinical application of therapeutics and simple video demonstrations followed by hands on training with manikins were found to be useful and definitely served towards improvement in performance.74\% felt that it covered the various must know aspects of therapeutics, $66 \%$ felt that it helped in the performance of theory exams and again $66 \%$ felt that it helped in relating knowledge to practice. It would prove to be more fruitful if a team involving pharmacologists and clinicians make an integrated approach to redefining the curriculum in pharmacology on critical areas of therapeutics. The involvement and motivated team work of concerned teaching faculty is also mandatory. ${ }^{11}$

Interestingly $81 \%$ of the students agreed that it helped in clarifying doubts better than lecture classes, $83 \%$ felt that it helped in the way of approach to patients while $89 \%$ agreed that it helped in performing well in the practical exams. $85 \%$ of the students agreed to the usefulness of the method. Thus the overall knowledge obtained in the second year curriculum could be better retained through the years of clinical course. A systematic and a meticulous rescheduling of the pharmacology curriculum covering the critical area of therapeutics is the need of the hour toward competency building skills so as to create confidence in clinical application skills. Various integrated medical curricula have been adopted by many medical schools all over the world to ensure holistic approach rather than a fragmented one which in turn encourages meaningful learning in medical education. ${ }^{12,13}$

The entire curriculum of pharmacology can be restructured uniformly into topics for large group discussions, topics for small group discussions, interpretational and performance based skill building exercises on the various practical must know topics as per the time slot allotted to the subject. The implementation of a revised curriculum is not easy. ${ }^{14,15}$ Steps towards development of a core concept exploring key areas of clinical pharmacology and therapeutics has always been the principle aim of undergraduate curriculum. The undergraduate pharmacology curriculum has always been a topic of intense debate. It has been generally felt that pharmacology course in medical schools has failed to keep pace with the rapid changes and requirements of clinical practice. Traditionally, it has focused more on factual information with little or no emphasis on clinical and applied aspects. ${ }^{16}$ Our student learning outcomes include the cognitive, psychomotor, and affective domains of learning as per the Bloom's cognitive taxonomy and competency levels. ${ }^{17}$

The limitation of the study is that it has been done in one center only. Even though the CRRI students were a step ahead in their clinical experience, the final year students were found to perform better in the study with respect to the knowledge in pharmacology. The difference in time gap after finishing pharmacology between the two groups, could have played a role in the better performance of the final year students as well. The lesson plans were entirely framed by pharmacologists.

Identifying the key areas of focus while revising the curriculum and method of teaching, is the top priority which should involve an expert team of clinicians, who can make the recommendations on the pitfalls on therapeutic application skills that are usually encountered among students during clinical classes, along with pharmacologists. A time slot on clinical pharmacology and rational therapeutics in the final year curriculum as a refresher course can be introduced focusing on prescribing skills, dose calculations, adverse drug reaction monitoring, antibiotic prescribing, on importance of monitoring therapeutic concentrations to name a few. It is perhaps not surprising that new doctors consider prescribing to be the most difficult aspect of their job and the one for which they feel least prepared. ${ }^{18}$ Prescribing forms a significant component of the work of newly qualified doctors and is probably the intervention that has the greatest influence on the health of their patients. ${ }^{19}$

\section{CONCLUSION}

The study has been planned and executed based on the small group discussions on must know topics on therapeutics and various skill development modules with the help of simulated models and appropriate OSPE stations that can help to improve the student's performance 
skills in a clinical setting. The retainment of knowledge gained has been assessed to be better and the changes made, has been found to have provoked interest and better insight into attitudinal skills of the students. We have to critically analyze and accept that the second year curriculum in pharmacology is only a sensitization program on various pharmacological aspects considering the vastness and factualness of the subject. It is highly impossible for the subject to be mastered in the one-year period and it is time to incorporate pharmacology and therapeutics in the final year curriculum involving the pharmacologists and a time slot for update on clinical pharmacology and rational therapeutics in the CRRI period.

\section{ACKNOWLEDGEMENTS}

Authors would like to render their sincere and heartfelt thanks to the students who actively and eagerly participated in the study and supported in completing this study. Also they would like to thank the central library facility and faculty of Stanley Medical College for the support.

\section{Funding: No funding sources}

Conflict of interest: None declared

Ethical approval: The study was approved by the Institutional Ethics Committee

\section{REFERENCES}

1. De Vires TO, Henning RH, Hogerzeil HV, Frwsle DA. Guide to good prescribing a practical manual. Geneva: World Health Organization; 1994.

2. Richir MC, Tichelaar J, Geijteman ECT, de Vries TPGM. Teaching clinical pharmacology and therapeutics with an emphasis on the therapeutic reasoning of undergraduate medical students. European Journal of Clinical Pharmacology. 2008;64(2):217-24.

3. Badyal DK, Bala S, Kathuria P. Student evaluation of teaching and assessment methods in pharmacology. Indian J Pharmacol. 2010;42:87-9.

4. Devi V, Bhat V, Shenoy GK. Undergraduate pharmacology curriculum at an international medical college in India. Indian J Pharmacol. 2016;48:S1:148.

5. Ian E.Hughe. Computer based learning-an aid to successful teaching of pharmacology? Naunyn Schmiedebergs Arch Pharmacol. 2002;366:77-82.

6. Govindaraja C, Jaiprakash H, Annamalai C, Vedhavathy SS. Computer assisted learning: Perceptions and knowledge skills of undergraduate medical students in a Malaysian medical school. Natl J Physiol Pharm Pharmacol. 2011;1:63-7.

7. Hughes IE. Do computer simulations of laboratory practicals meet learning needs? Trends Pharmacol Sci. 2001;22:71-4.
8. Rathnakar UP, Gopalakrishna HN, Pai PG, Ullal SD, Pemminati S, Pai MRSM, et al. Didactic lecture and interactive sessions in small groups: A comparative study among undergraduate students of pharmacology in India. JCDR. 2010;4(2):2260-64.

9. Pal R, Kar S, Zaman FA, Jha DK, Pal S. Assessment of Impact of Small Group Teaching Among Students in Community Medicine. Indian Journal of Community Medicine: Official Publication of Indian Association of Preventive and Social Medicine. 2012;37(3):170-3.

10. Dewhurst D. Computer-based alternatives in higher education past, present and future. ALTEX. 2006;23:197-201.

11. Peets AD, Coderre S, Wright B, et al. Involvement in teaching improves learning in medical students: a randomized cross-over study. BMC Medical Education. 2009;9:55.

12. Dubois EA, Franson KL. Key steps for integrating a basic science throughout a medical school curriculum using an e-learning approach. Med Teach. 2009;31:822-8.

13. Rao KH, Rao RH. Perspectives in medical education 5. Implementing a more integrated, interactive and interesting curriculum to improve Japanese medical education. Keio J Med. 2007;56:75-84.

14. Mehta P, Ingole N. Mechanics of paper setting: Being a paper setter. In: Pritha Bhuiyan P, Supe A, Rege N (eds). The Art of Teaching Medical Students, $3^{\text {rd }}$ Ed. India; Elsevier. 2015:276-287.

15. Desai M. Changing face of pharmacology practicals for medical undergraduates. Indian Journal of Pharmacology. 2009;41(4):151-2.

16. Dikshit RK. Rational education for rational therapy. World Health Forum.1992;13:151.

17. Anderson LW, Krathwohl DR, Airasian PW, Cruikshank KA, Mayer RE, Pintrich PR, et al. A Taxonomy for Learning, Teaching, and Assessing: A Revision of Bloom's Taxonomy of Educational Objectives, New York: Longman; 2001.

18. Illing JC, Morrow GM, Rothwell nee Kergon CR, et al. Perceptions of UK medical graduates' preparedness for practice: A multi-centre qualitative study reflecting the importance of learning on the job. BMC Medical Education. 2013;13:34.

19. Ross S, Maxwell S. Prescribing and the core curriculum for tomorrow's doctors: BPS curriculum in clinical pharmacology and prescribing for medical students. British Journal of Clinical Pharmacology. 2012;74(4):644-61.

Cite this article as: Rathinam J, Divakar R, Subahan SP, Preethi A, Vanitha G, Punitha R. A comparative assessment of the impact of computer assisted learning of knowledge and skill based competencies among undergraduate students in a medical college in South India. Int J Basic Clin Pharmacol 2018;7:612-6. 